\title{
The ATLAS New Small Wheel Simulation and Reconstruction Software and Detector Performance Studies
}

\section{Chara Kitsaki*, on behalf of the ATLAS Muon Collaboration}

National Technical University of Athens, Greece

E-mail: chara.kitsaki@cern.ch

In view of the Large Hadron Collider (LHC) luminosity upgrades, the New Small Wheels (NSW) will replace the present Small Wheel stations of the ATLAS end-cap Muon Spectrometer. The aim of the NSW is to maintain the same level of efficiency, momentum threshold and resolution in the expected higher background level and to keep an acceptable muon trigger rate. The NSW is equipped with two completely new detector technologies: the small-strip Thin Gap Chambers and the resistive-strip Micromegas. Currently the series production of the detectors is well advanced and the integration in sectors and assembly of the wheels is in progress. Quality control tests, including cosmic rays data taking are in full swing. The software for simulation and reconstruction of the NSW is also well advanced. The nominal geometries and misalignments are implemented, while the implementation of all other possible deviations from ideal operating conditions is ongoing. The detector's response simulation is underway with the aim to be compared with the real data collected from cosmic rays and test-beams. Finally, trigger and reconstruction performance studies are carried out in different configurations of the detectors and background levels. In this paper, an overview of the software implementation and the adopted strategies for simulation and reconstruction is presented. Furthermore, a summary of the real data studies for the future validation and comparison is given.

40th International Conference on High Energy physics - ICHEP2020

July 28 - August 6, 2020

Prague, Czech Republic (virtual meeting)

\footnotetext{
${ }^{*}$ Speaker
} 


\section{Introduction}

High Energy Physics relies on samples of simulated events, which provide a highly detailed and accurate simulation of the detector. The goal is to achieve a digitized simulation output that matches the data format recorded from the real collisions, such that the same reconstruction algorithms can be used on real and simulated data. The common framework used by the ATLAS experiment [1], is called Атнеna [2]. The standard simulation chain which is presented in Figure 1 can be summarized in the following steps:

- Event Simulation: The desired generated particles are transported through the detector volumes, while all the physics processes, i.e. interaction of particles with the detector materials, are simulated.

- Digitization: Simulation of the detector active area interactions and electronic signal formation. Particles interactions, i.e. energy deposits, positions, are translated into electronic signal.

- Reconstruction: The detector response (i.e. time response, spatial reconstruction) after the calibrations with the real data is reconstructed into objects used for the physics analysis.

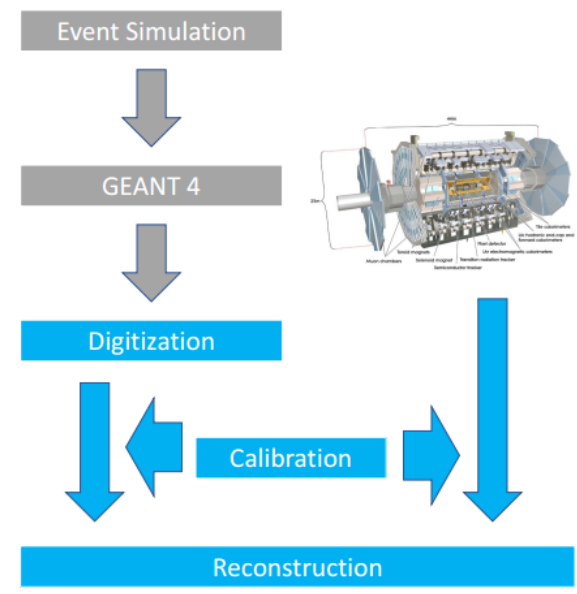

Figure 1: ATLAS simulation chain, from event simulation to reconstruction.

In order to efficiently handle the increased luminosity that will be provided by the LHC, the innermost stations of the ATLAS end-cap Muon Spectrometer will need to be replaced. Thus, the new detector technologies, namely the resistive-strip Micromegas (MM) and the small-strip Thin Gap Chambers (sTGC) need to be included in the full simulation chain.

\section{Detector Description}

A well-defined geometry description is a prerequisite for the simulation procedure. All the primary numbers are being collected from a database ${ }^{1}$ and a transient geometry representation is

\footnotetext{
${ }^{1}$ Oracle is used as the adopted solution.
} 
translated into Geant4 geometry. Due to schedule uncertainties, two possible scenarios have been accommodated for the ATLAS geometry, including the two new wheels on both end-caps or only the one on one side while maintaining the old wheel on the other side.

As the geometry is built at run-time, standalone visualization tools [3] have been developed, easing the validation and debugging, i.e. detection of overlapping volumes.

To emulate the detector response and reconstruct trajectories, the detailed description of the detectors' inner structure or in other words the active areas (the area where the detector physics takes place), is of high importance. For the sTGCs, the layers of pads, wires and strips and for the MM the strips layers have been implemented following each specific chamber's design characteristics. However, at the construction sites, all the permanent deformations are being described through detailed models with the aim to define the realistic description of the nominal geometry.

For the accurate localization of the muon tracks, all the possible misaligned and deformed states due to the special conditions in the cavern, should also be accounted. Time-dependent measurements provided by the optical alignment system [4], will be continuously monitored and used to reconstruct the geometry. The relative positions and rotations with respect to the initial, as well as the set of parameters describing the deformation and expansion states will be provided online by the independent alignment software [5] through the conditions database. ${ }^{2}$ These specific implementations are still under development.

\section{Digitization \& Reconstruction}

Simulation of the particle interaction and transportation within the detector active volume is digitized into signals as expected from the detector. The conversion is based on results taken from analytical simulations and/or from test beams then implemented as parameters or distributions. During this step, the detector noise and pileup events are overlaid.

For the track reconstruction, local pattern recognition algorithms are used to identify the track segments while a track fit is performed resulting in the muon track candidates. All the various techniques adapted from the test beams analyses during the R\&D period have been implemented and are under optimization using as reference results from real data.

\section{Trigger Simulation}

ATLAS uses a two level system [6] for event selection and recording. The Level-1 trigger is hardware based and preselects the candidate events. The discrimination against backgrounds is performed by combining track segments found in the various subsystems. The accepted event information is passed to the software based high level trigger stage. Studies with collision data showed more than $90 \%$ mismatches between the Level-1 triggered and the offline reconstructed muons (see Figure 2 for 2017 data). While at luminosities so far achieved by LHC this dominant fake trigger was sustainable, this will not be the case any longer for higher luminosities. Therefore the main focus of the Muon Spectrometer is to improve the rejection of fake triggers. This will be achieved with the NSW which is designed to provide an angular resolution with the accuracy of 1 mrad [9]. By combining the segments coming from the middle layer (Big Wheel) of the Muon

\footnotetext{
${ }^{2}$ updated every few hours
} 
Spectrometer with the segment angles provided by the NSW, the background is expected to be effectively suppressed.

In Figure 3 the transverse momentum distribution of the offline reconstructed muon candidates is shown. The candidates' threshold is set at $20 \mathrm{GeV}$. When enabling the coincidences from the NSW in simulation, in the low $p_{\mathrm{T}}$ regions the fake candidates are reduced.

In Figure 2 the rejection efficiency of each subsystem is illustrated overall in the ATLAS detector. The NSW covers the $1.3<\eta<2.4$ region and the estimated rejection including it into the coincidence is shown. It is visible that a $\sim 45 \%$ fake reduction can be achieved. The expected distribution of the muon candidates in Run 3, shown on Figure 2, results from a perfect matching with the muons which are reconstructed by the offline algorithm.

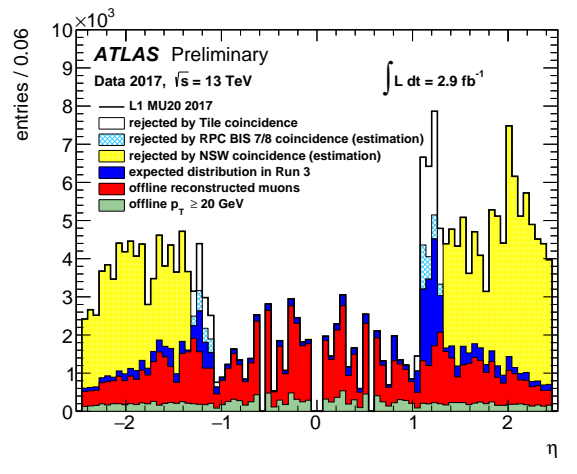

Figure 2: $\quad \eta$ distribution of L1_MU20 muon candidates [7] in 2017 data.

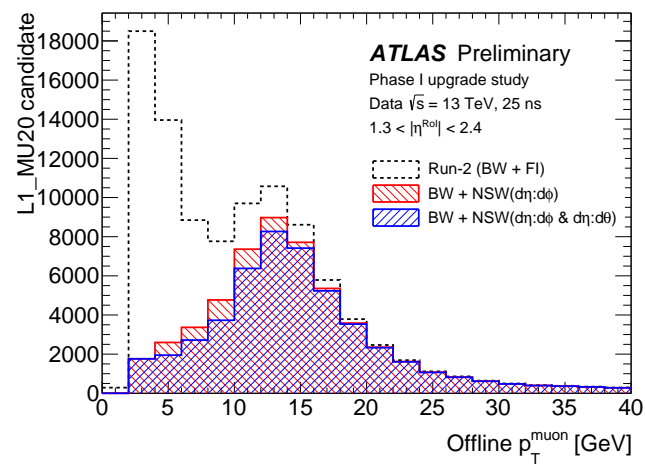

Figure 3: $p_{\mathrm{T}}$ distribution of L1_MU20 muon candidates [7]. In the low $p_{\mathrm{T}}$ region the fake candidates are rejected effectively when including each NSW coincidence logic.

\section{Detector performance}

Before the installation of the detector assemblies on the wheels, the chambers need to pass a sequence of QA/QC criteria in order to ensure that the system works within the specifications.

During the quadruplet construction, gas leaks and high voltage instabilities need to be identified and fixed if possible as after the installation such kind of problems should be unrecoverable.

After this step, the integration and validation of the electronics and of the alignment system is performed in the surface facilities at CERN. Once completely assembled and before the placement on the wheels, the chambers undergo tests with cosmic rays. ${ }^{3}$ The efficiency maps and position resolution measurements can give an overview of the detector status and performance.

Some of the performance plots have been collected and are shown in Figures 4, 5 for MM and sTGC respectively. For different MM high voltage configurations the efficiency is recorded to qualify the final integration with the sTGC. At the nominal working point of $570 \mathrm{~V}$ the MM layers reach an efficiency of $\sim 95 \%$. The hits on the readout elements are grouped in clusters. At large

\footnotetext{
${ }^{3}$ Micromegas only. sTGC cosmic ray tests are part of the production QA/QC (at the construction sites). They cannot be performed in the CERN surface facility as the necessary safety equipment is available only inside the cavern.
} 

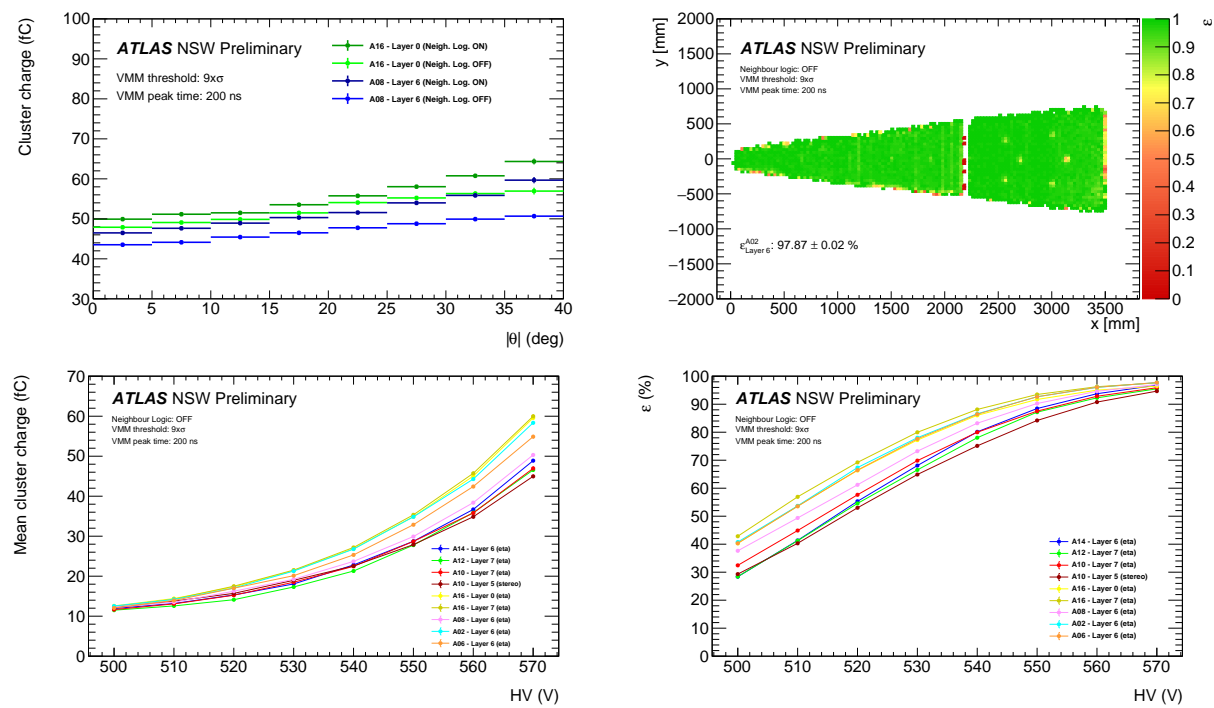

Figure 4: Micromegas performance with cosmic muons [8]. Cluster charge and efficiency studies. The differences in efficiency between the layers are probably coming from the observed variation in gain (effect under investigation).

incident angles, more strips contribute in the clustering algorithm, thus the charge is expected to be raised compared to small angles. NSW software aims to collect and use results from cosmic tests and/or from test-beams (for sTGC) for comparisons and validation of the simulations.
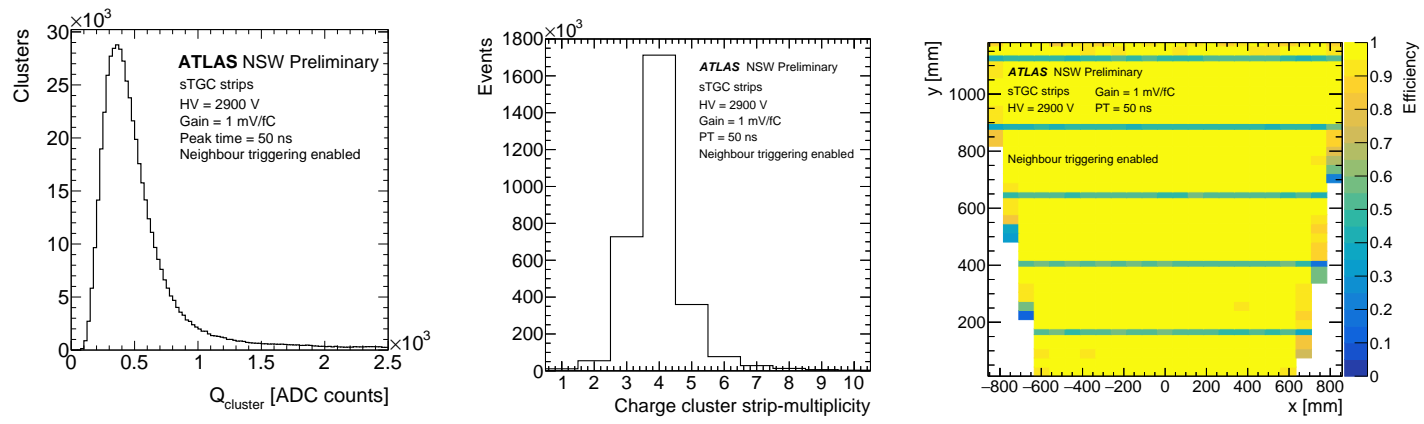

Figure 5: sTGC performance results [8] from cosmic tests at construction site. Measurements on the cluster charge, multiplicity and efficiency of a strip layer.

\section{Conclusions}

The NSW implementation in the common ATLAS software is advancing well. The geometries have been implemented while all the corrections which reflect the realistic layout soon will be finalized. In parallel, the validation of the implementation is performed with the independent alignment software output. All the clusterization algorithms for the track reconstruction are already included and calibration tools are continuously updated and optimized with the aim to use the same configurations and achieve the same performance of test beams and cosmic tests. The NSW is 
included in the trigger algorithm proving the desired fake suppression in the regions of interest according to the requirements [9]. The complete analysis chain with the NSW in place will be ready for use for the upcoming LHC Run 3 data-taking period.

\section{Acknowledgments}

This work was supported by the Hellenic Foundation for Research and Innovation (HFRI) under the HFRI PhD Fellowship grant (Fellowship Number: 800).

\section{References}

[1] ATLAS Collaboration, The ATLAS Experiment at the CERN Large Hadron Collider, JINST 3 (2008) S08003.

[2] The Athena Framework, https://atlassoftwaredocs.web.cern.ch/athena/athena-intro.

[3] M. Bandieramonte et al., FullSimLight: ATLAS standalone Geant4 simulation in proceedings of 24th International Conference on Computing in High Energy and Nuclear Physics, Adelaide, Australia, 4 - 8 Nov 2019, ATL-SOFT-PROC-2020-023, https://cds.cern.ch/record/2712863.

[4] C. Amelung et al., The ATLAS muon alignment system in proceedings of the first LHC Detector Alignment Workshop, CERN, Geneva, Switzerland, 4-6 Sept 2006, 10.5170/CERN2007-004.159, https://cds.cern.ch/record/1047115.

[5] C. Amelung, ARAMyS - Alignment Reconstruction Software for the ATLAS Muon Spectrometer, Nuclear Physics B (Proc. Suppl.) 172 (2007) 132-134.

[6] ATLAS Collaboration, Performance of the ATLAS trigger system in 2015, Eur. Phys. J. C. 77 (2017) 317.

[7] ATLAS Collaboration, L1 Muon Trigger Public Results, https://twiki.cern.ch/twiki/bin/view/AtlasPublic/L1MuonTriggerPublicResults.

[8] ATLAS Collaboration, NSW Public Results, https://twiki.cern.ch/twiki/bin/view/AtlasPublic/NSWPublicResults.

[9] ATLAS Collaboration - Technical Design Report - New Small Wheel. CERN-LHCC-2013006, ATLAS-TDR-020, https://cds.cern.ch/record/1552862. 